
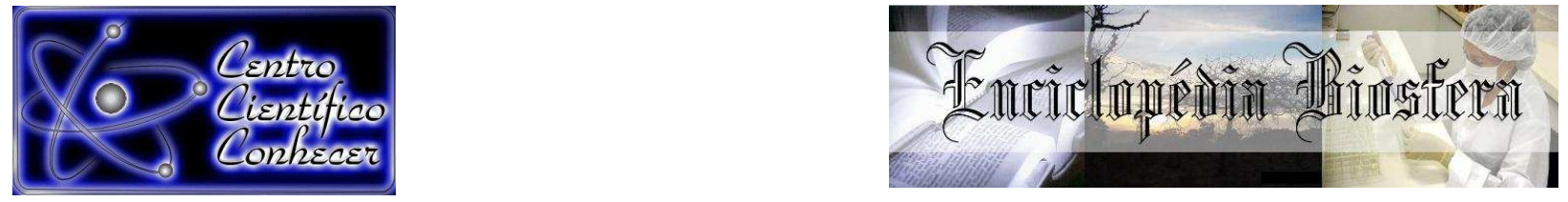

\title{
PERFIL DA MORBIDADE HOSPITALAR POR ACIDENTES DE TRANSPORTE NO MUNICÍPIO DE RIO BRANCO-ACRE, BRASIL, NO PERÍODO DE 2014 E 2015
}

Tauan de Lima Ramos $^{1}$, Airton Lima de Aguiar ${ }^{1}$, Eder Ferreira de Arruda $^{2}$ Luciana Ribeiro da Silva Peniche ${ }^{2}$ Cristiane Santos de Sousa ${ }^{2}$

1. Graduando em Fisioterapia pela União Educacional do Norte (UNINORTE), Rio Branco - AC (tauan_lima@hotmail.com)

2. Docente do curso de Fisioterapia da União Educacional do Norte (UNINORTE), Rio Branco - AC

Recebido em: 03/10/2016 - Aprovado em: 21/11/2016 - Publicado em: 05/12/2016 DOI: 10.18677/EnciBio_2016B_135

\section{RESUMO}

Os acidentes de transporte (AT) configuram-se como uma importante causa externa de internações hospitalares. Esse trabalho teve como objetivo investigar o perfil da morbidade hospitalar por acidentes de transporte em Rio Branco-Acre no período de 2014 e 2015. Trata-se de um estudo transversal realizado a partir de dados de domínio público referentes internações hospitalares por AT coletados no Sistema de Informação Hospitalar SIH/SUS. Entre 2014 e 2015, foram registradas 1565 internações por AT, destas, 739 ocorreram em 2014 e 826 em 2015. O maior número de internações por AT foi verificado nos homens, sendo 573 (78\%) em 2014 e 670 (81\%) em 2015. A faixa etária de 20 e 39 anos apresentou maior frequência de internações por AT totalizando 793 casos, sendo 371 (50\%) em 2014 e 422 (51\%) em 2015 . Os meses de fevereiro (3,28\%), junho $(3,08 \%)$ e outubro $(2,98 \%)$ em 2014 e janeiro (3,94\%), fevereiro (3,13\%) e outubro (3,59\%) em 2015 obtiveram as maiores proporções de hospitalizações por AT, enquanto o mês de julho obteve as menores proporções no biênio analisado. Em 2014, 100\% das hospitalizações foram realizadas em hospitais públicos, enquanto em 2015 foram registradas $85 \%$ das internações em hospitais públicos e 15\% em hospitais privados. Mais da metade das internações por AT ocorreram com indivíduos que transitavam em motocicletas, sendo $61 \%$ em 2014 e 60\% em 2015. Dado o exposto, conclui-se que os AT são um problema prioritário de saúde em Rio Branco-AC, assim necessitando da realização de políticas públicas visando à mudança dessa situação.

PALAVRAS-CHAVE: hospitalizações, SUS, Trânsito.

\section{PROFILE THE HOSPITAL MORBIDITY DUE TO TRANSPORTATION ACCIDENTS IN MUNICIPALITY OF RIO BRANCO-ACRE, BRAZIL, DURING 2014 AND 2015}

\begin{abstract}
The traffic accidents (TA) are configured as an important external cause of hospital admissions. This study aimed to investigate the profile of hospital morbidity from transport accidents in Rio Branco-Acre in 2014 and 2015. This search is a crosssectional study conducted from public domain data on hospital admissions for AT collected System Hospital Information SIH / SUS. Between 2014 and 2015, 1565
\end{abstract}


hospital admissions were recorded by AT, of these, 739 occurred in 2014 and 826 in 2015. The highest number of hospitalizations for AT was found in men, and 573 (78\%) in 2014 and 670 (81\%) in 2015. The age range of 20 and 39 years showed higher frequency of hospitalizations for AT totaling 793 cases, and 371 (50\%) in 2014 and $422(51 \%)$ in 2015 . The months of February (3.28\%), June (3.08\%) and October $(2.98 \%)$ in 2014 and January (3.94\%), February $(3.13 \%)$ and October $(3.59 \%)$ in 2015 obtained the highest rates of hospitalizations for AT while July obtained the lowest proportions in the analyzed biennium. In 2014, $100 \%$ of hospitalizations were performed in public hospitals, while in 2015 were recorded $85 \%$ of hospitalizations in public hospitals and $15 \%$ in private hospitals. More than half of hospitalizations for AT occurred with individuals who transited on motorcycles, and $61 \%$ in 2014 and $60 \%$ in 2015 . Given the above, it is concluded that the AT is a priority health problem in Rio Branco-AC, thus necessitating the implementation of public policies aimed at changing this situation.

KEYWORDS: Traffic, hospitalizations, SUS.

\section{INTRODUÇÃO}

Morbidade refere-se ao número de indivíduos de uma população atingidos por uma determinada doença ou agravo à saúde. Os padrões de morbidade de uma população podem ser mensurados através das internações hospitalares, denominado assim de morbidade hospitalar (LEBRÃO et al., 1997).

No Brasil, as causas externas são os principais e mais importantes motivos de morbidade hospitalar. JORGE \& KOIZUMI (2004) relatam que os gastos do SUS com as internações hospitalares por causas externas são superiores aos gastos com hospitalizações por causas naturais.

As principais causas externas de morbidade hospitalar englobam afogamentos, envenenamentos, quedas, agressões, homicídios, suicídios, abusos sexuais, assim como as lesões decorrentes dos acidentes de transporte (LIGNANI \& VILLELA, 2013). Segundo SOUZA et al. (2007) AT é um evento não intencional de origem multifatorial, porém evitável.

Os AT representam um importante problema social e de saúde pública e constituem-se como uma das mais relevantes causas externas de lesões, fraturas e principalmente de internações hospitalares e óbitos. Segundo SOARES \& BARROS (2006) os AT representam mais de um quarto das mortes violentas do país e respondem por $20 \%$ das internações, ocupando o segundo lugar no conjunto das causas externas de internações hospitalares.

No ambiente hospitalar, o indivíduo vítima de AT dispõe de atendimento clínico e cirúrgico essencial à manutenção da vida, bem como deve receber atendimento fisioterapêutico visando à reabilitação, pois é necessário restaurar a funcionalidade e integridade física que tenham sido comprometidas em decorrência do acidente (VIEIRA et al., 2010).

Neste contexto, o conhecimento dos índices das internações hospitalares devido aos AT no município de Rio Branco-Acre é essencial para que se tenham informações precisas da frequência dessa condição mórbida visando o direcionamento de ações preventivas. Dado o exposto, se faz necessário à realização da presente pesquisa que tem como objetivo investigar o perfil da morbidade hospitalar por AT em Rio Branco-Acre no período de 2014 e 2015. 


\section{MATERIAL E MÉTODOS}

O presente estudo trata-se de um estudo descrito transversal. Segundo HOCHMAN et al., (2005) os estudos transversais são de grande utilidade para avaliação da situação de saúde de uma população e possuem como principais vantagens o fato de serem de baixo custo e tempo de execução. A população de estudo foi composta por todos os casos de hospitalizações por AT ocorridos e registrados no município de Rio Branco-Acre de janeiro de 2014 a dezembro de 2015.

Os dados referentes internações hospitalares por AT foram coletados a partir de informações do Sistema de Informação Hospitalar SIH/SUS disponíveis no sítio eletrônico do Departamento de Informática do Sistema Único de Saúde (DATASUS). Foram considerados como casos de acidentes de trânsito terrestre, todos os registros classificados pelo diagnóstico secundário que utilizavam os códigos (V01V89) do capítulo XX da Décima Revisão da Classificação Estatística Internacional de Doenças e Problemas Relacionados à Saúde (CID-10).

Calculou-se a proporção percentual de internações hospitalares, da seguinte forma:

\section{Proporção $=($ № de internações hospitalares por acidentes de transporte terrestre / № total de internações hospitalares) x 100}

A proporção percentual de internações hospitalares foi calculada segundo meses do ano (janeiro-dezembro), tipo de hospital (público; particular) e tipo de transporte (automóvel, motocicleta, bicicleta, pedestre e demais tipos). A presente proposta de pesquisa não foi submetida ao Comitê de Ética em Experimentação com Seres Humanos, pois foram utilizados exclusivamente dados de acesso público, sem a identificação dos pacientes internados.

\section{RESULTADOS E DISCUSSÃO}

Entre 2014 e 2015, no município de Rio Branco-AC foram registradas 55.302 internações hospitalares, sendo 27.308 em 2014 e 27.994 em 2015. Nesse mesmo período, o total de hospitalizações por AT somou 1.565 internações, sendo registradas 739 em 2014 e 826 em 2015.

Com relação ao sexo, o maior número de internações por AT foi verificado nos indivíduos do sexo masculino 573 (78\%) em 2014 e 670 (81\%) em 2015 (Tabela 1). Dados semelhantes foram encontrados por LIGNANI \& VILLELA (2013) no estudo descritivo sobre as internações por causas externas no município de Belo Horizonte-MG, no período de 2008 a 2010 onde o maior número de internações por AT ocorreu entre os homens com um total de 4.732 hospitalizações no período analisado.

MAGALHÃES et al., (2011) ao realizarem um estudo transversal de base populacional no período de 2007 a 2008, nas zonas urbana e rural de Rio BrancoAC sobre a prevalência autorreferida de AT identificaram maior número de AT nos indivíduos do sexo masculino com maior probabilidade de acidentes entre os indivíduos que costumam ingerir bebida alcoólica.

Segundo PANICHI \& WAGNER (2006), a predominância do sexo masculino em AT pode ser atribuída a uma maior exposição dos homens aos comportamentos de risco para acidentes os quais incluem 0 fato dos homens serem predominantemente os condutores e por ingerirem bebida alcoólica previamente à direção de veículo. 
Dentre as faixas etárias, os indivíduos entre 20 e 39 anos apresentaram maior frequência de internações por AT totalizando 793 casos, sendo 371 (50\%) em 2014 e $422(51 \%)$ em 2015 (Tabela 1). Resultado parecido foi verificado por CAIXETA et al., (2010) que ao realizarem um transversal nos anos de 2005 e $2006 \mathrm{com}$ o objetivo de descrever o perfil das vítimas e circunstâncias dos AT em residentes da cidade de Goiânia-GO, identificaram maior porcentagem de indivíduos acima de 18 anos de idade. Semelhantemente, MAGALHÃES et al., (2011) no município de Rio Branco-AC identificou a faixa etária dos 18 aos 25 anos como a faixa etária com maior prevalência de AT.

De acordo com CHAGAS (2011), a inexperiência para dirigir é um fator contribuinte de acidentes de trânsito urbano e essa é uma característica comum entre os condutores mais jovens. O relatório de saúde mundial publicado pela OMS em 2002 apontou que os indivíduos entre 15 e 44 anos foram responsáveis por mais da metade de todas as mortes no trânsito no referido ano (WORLD HEALTH ORGANIZATION, 2002).

TABELA 1 - Frequência de casos de internação hospitalar por AT, segundo sexo e faixa etária da população de Rio Branco-Acre no biênio (2014-2015).

\begin{tabular}{lcc}
\hline \multirow{2}{*}{ Variáveis } & \multicolumn{2}{c}{ Frequência de Internações } \\
& $\mathbf{n}(\%)$ \\
\cline { 2 - 3 } & $\mathbf{2 0 1 4}$ & $\mathbf{2 0 1 5}$ \\
\hline Sexo & $573(78)$ & $670(81)$ \\
Fasculino & $166(22)$ & $156(19)$ \\
Faixa etária & & \\
0 a 9 anos & $22(3)$ & $34(4)$ \\
10 a 19 anos & $124(17)$ & $102(12)$ \\
20 a 39 anos & $371(50)$ & $422(51)$ \\
40 a 59 anos & $178(24)$ & $211(26)$ \\
60 anos e mais & $44(6)$ & $57(7)$ \\
Total & $739(100)$ & $826(100)$ \\
\hline
\end{tabular}

Fonte: DATASUS, 2016.

No que se referem aos meses do ano, fevereiro $(3,28 \%)$, junho $(3,08 \%)$ e outubro $(2,98 \%)$ em 2014 e janeiro $(3,94 \%)$, fevereiro $(3,13 \%)$ e outubro $(3,59 \%)$ em 2015 obtiveram as maiores proporções de hospitalizações por AT, enquanto o mês de julho obteve as menores proporções no biênio analisado, sendo 1,82\% e 2,30\% em 2014 e 2015, respectivamente (Figura 1).

Com relação ao mês de outubro, o presente estudo apesar de ter encontrado valores menores corrobora com os resultados obtidos por ANDRADE et al., (2011) que verificaram que $11,59 \%$ dos 3.159 AT fatais na rodovia federal do Estado do Pará em 2008 ocorreram no referido mês. Já em relação ao mês de fevereiro, este estudo encontrou resultado diferente do identificado pelos autores supracitados, pois os mesmos verificaram o mês de fevereiro com o menor índice de acidentes. Porém, o mês de julho tanto neste estudo como no estudo de ANDRADE et al., (2011) apresentou as menores proporções de AT.

SOARES \& BARROS (2006), ao analisarem os fatores associados ao risco de internação da coorte constituída por 3.468 vítimas dos AT ocorridos no Município de Maringá-PR, no ano 2000 , evidenciaram que a maioria dos acidentes ocorreu no $4^{\circ}$ 
trimestre do ano, com um total de 203 (30,2\%) de internações.

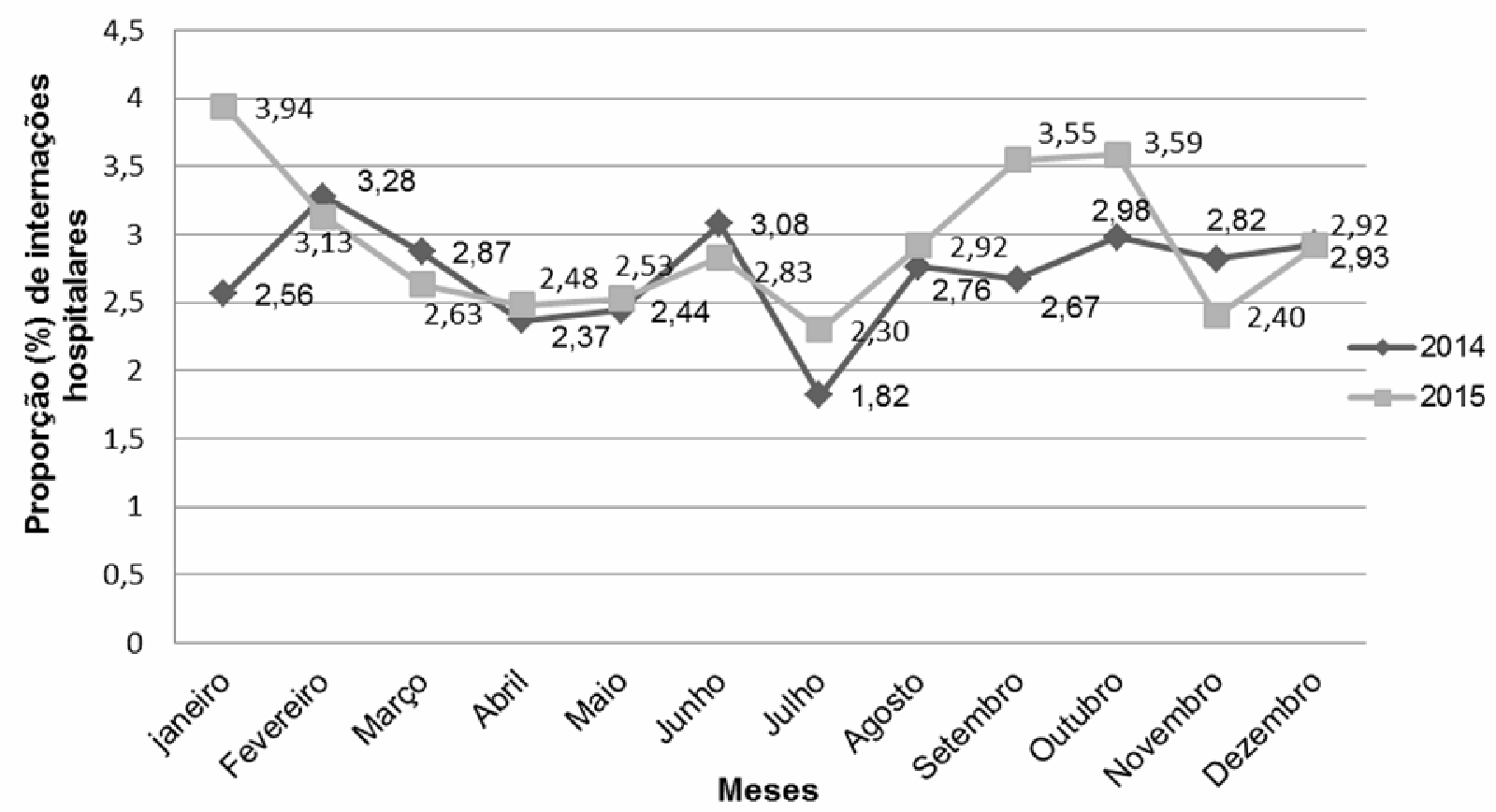

Fonte: DATASUS, 2016.

FIGURA 1 - Proporção (\%) de internações hospitalares por AT, segundo os meses dos anos em Rio Branco-Acre no biênio (2014 -2015).

No que diz respeito ao tipo de hospital no qual foram efetivadas as internações, verificou-se que em 2014 todas as hospitalizações por AT, 729 (10,0\%) foram realizadas em hospitais públicos, enquanto em 2015 foram registradas 702 (85\%) internações por AT em hospitais públicos e 124 (15\%) em hospitais privados, os quais atendem pelo SUS de forma complementar. A proporção de internações em hospitais públicos em 2014 foi de 2,70\%, já em 2015 a proporção foi de 2,50\% em hospitais públicos e de $0,44 \%$ em hospitais privados (Tabela 2).

Segundo COELHO \& ISTAKE (2014) as internações por acidentes de transporte geram elevados gastos públicos em saúde, sendo o segundo principal responsável por gastos dentre as internações por causas externas, prioritariamente em hospitais públicos. Quanto ao tipo de transporte, mais da metade das internações por AT ocorreram com indivíduos que transitavam em motocicletas, sendo 454 (61\%) em 2014 e 496 (60\%) em 2015. A proporção de hospitalização por AT em 2014 foi de 1,66\% e em 2015 chegou a 1,77\% (Tabela 2).

ROCHA (2010) ao estudar os acidentes de trânsito no município de Rio Branco-AC, no período de janeiro de 2005 a dezembro de 2008 verificou que o tipo de veículo mais envolvido em acidentes foi à motocicleta $(43,4 \%)$. Em outro estudo transversal realizado por ROCHA \& SCHOR (2013) foram observados 3.582 acidentes com motocicleta e 3.768 vítimas em Rio Branco-AC. Segundo os autores a frota de motocicletas teve um crescimento de $72,8 \%$, com uma taxa de envolvimento em acidentes com um aumento de $42,2 \%$, fato este que pode explicar os elevados índices de acidentes e internações entre os indivíduos que conduzem este tipo de veículo.

Outros estudos observaram também a predominância de acidentes e hospitalizações de indivíduos que conduziam motocicletas. Na pesquisa realizada 
por MASCARENHAS \& BARROS (2015), com o objetivo de descrever as características das internações hospitalares por causas externas no sistema público de saúde do Brasil em 2011, encontraram que das 973.015 internações, predominaram as internações por quedas $(38,4 \%)$ e acidentes de transporte terrestre (15,8\%), sendo que das internações por AT, 77.595 casos ocorreram com motociclistas, com uma média de 6,1 dias de internação e 2,3\% de letalidade.

No estudo desenvolvido por GORIOS et al., (2015) com o objetivo de caracterizar os acidentes de motocicleta ocorridos na cidade de São Paulo-SP no ano de 2013 identificou-se que ocorreram 5.597 internações de motociclistas traumatizados em acidentes de transporte, sendo $89,8 \%$ do sexo masculino. De forma semelhante, BARBOSA et al., (2014) ao analisarem, no ano de 2009, 430 fichas de atendimento a vítimas de acidentes de motocicleta, observaram que $73,5 \%$ das vítimas eram do sexo masculino.

TABELA 2 - Frequência e proporção (\%) de internações hospitalares por AT, segundo tipo de hospital e meios de transporte em Rio Branco-Acre no biênio (2014-2015).

\begin{tabular}{|c|c|c|c|c|}
\hline \multirow[t]{2}{*}{ Variáveis } & \multicolumn{2}{|c|}{$\begin{array}{l}\text { Frequência de internações } \\
\text { n (\%) }\end{array}$} & \multicolumn{2}{|c|}{$\begin{array}{c}\text { Proporção de internações } \\
(\%)\end{array}$} \\
\hline & 2014 & 2015 & 2014 & 2015 \\
\hline \multicolumn{5}{|c|}{ Tipo de hospital } \\
\hline Público & $739(100)$ & $702(85)$ & 2,70 & 2,50 \\
\hline Particular & $00(00)$ & $124(15)$ & 0,0 & 0,44 \\
\hline \multicolumn{5}{|c|}{ Meio de transporte } \\
\hline Automóvel & $19(3)$ & $22(3)$ & 0,06 & 0,07 \\
\hline Motocicleta & $454(61)$ & $496(60)$ & 1,66 & 1,77 \\
\hline Bicicleta & $57(8)$ & $61(7)$ & 0,20 & 0,21 \\
\hline Pedestre & $89(12)$ & $66(8)$ & 0,32 & 0,23 \\
\hline Outros & $120(16)$ & $181(22)$ & 0,43 & 0,64 \\
\hline
\end{tabular}

Fonte: DATASUS, 2016.

\section{CONCLUSÕES}

Os resultados deste estudo apontam os acidentes de transporte como um problema prioritário de saúde em Rio Branco-AC, principalmente entre os indivíduos do sexo masculino, na faixa etária de 20 a 39 anos e os motociclistas, pois são os principais responsáveis por grande parte das internações hospitalares. Dado o presente estudo, verifica-se que há necessidade de criação e direcionamento de ações preventivas para a mudança dessa situação e o desenvolvimento de novas pesquisas que investiguem os fatores de risco e causais dos acidentes.

\section{REFERÊNCIAS}

ANDRADE, E. M.; RAMOS, E. M. L. S.; ALMEIDA, S. S.; ARAÚJO, A. R.; PINHEIRO, W. R. F. Abordagem estatística dos acidentes de trânsito fatais ocorridos em rodovia federal do Estado do Pará. Revista Brasileira de Segurança Pública, ano 5, 9 ed., p.134-151, 2011 134-151. Disponível em: <http://revista.forumseguranca.org.br/index.php/rbsp/article/view/102/99>. 
BARBOSA, M. Q.: ABRANTES, K. S. M.; SILVA JÚNIOR, W. R.; CASIMIRO, G. S.; CAVALCANTI, A. L. Acidente Motociclístico: Caracterização das Vítimas Socorridas pelo Serviço de Atendimento Móvel de Urgência (SAMU). Revista Brasileira de

Ciências da Saúde, v.18, n.1, p.3-10, 2014. Disponível em: <http://periodicos.ufpb.br/ojs/index.php/rbcs/article/view/12915>.

CAIXETA, C. R.; MINAMISAVA, R.; OLIVEIRA, L. M. A. C.; BRASIL, V. V. Morbidade por acidentes de transporte entre jovens de Goiânia, Goiás. Ciência \& Saúde Coletiva, v.15, n.4, p.2075-2084, 2010. Disponível em: <http://dx.doi.org/10.1590/S1413-81232010000400021>.

CHAGAS, D. M. Estudo sobre fatores contribuintes de acidentes de trânsito urbano. 2011. 114 p. Dissertação (Mestrado em Engenharia de Produção) Universidade Federal do Rio Grande do Sul, Porto Alegre-RS, Brasil. Disponível em: http://hdl.handle.net/10183/32553. Acesso em: 17 set. 2016.

COELHO, B.; ISTAKE, M. Gastos públicos com acidentes de transporte no Brasil e no Paraná. In: 11ํㅡㄹ Encontro de Economia Paranaense (Anais Eletrônicos), FECEA, Apucarana-PR, 2014. Disponível em: http://www.fecea.br/ecopar/uploads/19-11-14acidente\%20transporte.pdf. Acesso em: 30 set. 2016.

GORIOS, C.; ARMOND, J. E.; RODRIGUES, C. L.; PERNAMBUCO, H.; IPORRE, R. O.; COLOMBO-SOUZA, P. Análise das internações ocorridas por acidentes com motociclistas na cidade de São Paulo. Acta Ortopédica Brasileira, v. 23, n. 4, 212214, 2015. Disponível em:<http://dx.doi.org/10.1590/1413-78522015230400999>.

HOCHMAN, B.; NAHAS, F. X.; OLIVEIRA FILHO' R. S.; FERREIRA, L. M. Desenhos de pesquisa. Acta Cirúrgica Brasileira, v.20, n.2, p.1-9, 2005. Disponível em: <http://dx.doi.org/10.1590/S0102-86502005000800002>.

JORGE, M. H. P. M.; KOIZUMI, M. S. Gastos governamentais do SUS com internações hospitalares por causas externas: analise no Estado de São Paulo, 2000. Revista Brasileira de Epidemiologia, v.7, n.2, p.228-238, 2004. Disponível em: <http://dx.doi.org/10.1590/S1415-790X2004000200012>.

LEBRÃO, M. L.; JORGE, P. M.; LAURENTI, R. Morbidade hospitalar por lesões e envenenamentos. Revista de Saúde Pública, v.4, n.27, p.26-37, 1997. Disponível em:<http://dx.doi.org/10.1590/S0034-89101997000500003>.

LIGNANI, L. O.; VILLELA, L. C. M.; Estudos descritivos sobre a morbidade hospitalar por causas externas em Belo Horizonte, Estado de Minas Gerais, Brasil, 2008 2010. Epidemiologia e Serviço de Saúde, v.22, n.2, p. 225-334, 2013. Disponível em:<http://dx.doi.org/10.5123/S1679-49742013000200004>.

MAGALHÃES, A. F; LOPES, C. M.; KOIFMAN, R. J.; MUNIZ, P. T. Prevalência de acidentes de trânsito auto-referidos em Rio Branco, Acre 2011. Revista de Saúde Pública, v.45, n.4, p.738-744, 2011. Disponível em:< http://dx.doi.org/10.1590/S0034-89102011005000031>. 
MASCARENHAS, M. D. M.; BARROS, M. B. A. Caracterização das internações hospitalares por causas externas no sistema público de saúde, Brasil, 2011. Revista Brasileira de Epidemiologia, v. 18, n. 4, p. 771-784, 2015. Disponível em: <http://dx.doi.org/10.1590/1980-5497201500040008>.

PANICHI, R. M. D.; WAGNER, A. Comportamento de risco no trânsito: Revisando a literatura sobre as variáveis preditoras da condução perigosa na população juvenil. Revista Interamericana de Psicologia, v.40, n.2, p.159-166, 2006. Disponível em:< http://www.redalyc.org/articulo.oa?id=28440203>.

ROCHA, G. S. Acidentes de trânsito com vítimas no município de Rio Branco - Acre: perfil epidemiológico. Revista ABRAMET, v.28, n.1, p.47-54, 2010. Disponível em:<

ROCHA, G. S.; SCHOR, N. Acidentes de motocicleta no município de Rio Branco: caracterização e tendências. Ciência \& Saúde Coletiva, v.18, n.3, p.721-731, 2013. Disponível em:<http://dx.doi.org/10.1590/S1413-81232013000300018>.

SOARES, D. F. P.; BARROS, M. B. A. Fatores associados ao risco de internações por acidentes de trânsito no município de Maringá - PR. Revista Brasileira de Epidemiologia, v.2, p.193-205, 2006. Disponível em:< http://dx.doi.org/10.1590/S1415-790X2006000200006>.

SOUZA, M. F. M.; MALTA, D. C.; CONCEIÇÃO, G. M. S; SILVA, M. M. A.; GAZALCARVALHO, C.; NETO, O. L. M. Análise descritiva e de tendência de acidentes de transporte terrestre para políticas sociais no Brasil. Epidemiologia e Serviço de Saúde, v.16, n.1, p. 33-44, 2007. Disponível em:<http://dx.doi.org/10.5123/S167949742007000100004>.

VIEIRA, L. J. E. S.; SOUZA, E. R.; XAVIER, E. P.; LIRA, S. V. G.; FERREIRA, R. C. Relatos da equipe de saúde quanto às práticas educativas ao vitimado no transito durante a hospitalização/reabilitação num hospital de emergência. Saúde e Sociedade, v.19, n.1, p.213-223, 2010. Disponível em:< http://dx.doi.org/10.1590/S0104-12902010000100018>.

WORLD HEALTH ORGANIZATION. The World Health Report 2002: reducing risks, promoting healthly life. Geneva; 2002. Disponível em: http://www.who.int/whr/2002/en/. Acesso em: 16 set.2016. 